\title{
RAPID: Shrinking the Congestion-control Timescale
}

\author{
Vishnu Konda and Jasleen Kaur \\ University of North Carolina at Chapel Hill
}

\begin{abstract}
TCP congestion-control is fairly inefficient in achieving high throughput in high-speed and dynamic-bandwidth environments. The main culprit is the slow bandwidth-search process used by TCP, which may take up to several thousands of round-trip times (RTTs) in searching for and acquiring the end-to-end spare bandwidth. Even the recently-proposed "highspeed" transport protocols may take hundreds of RTTs for this.

In this paper, we design a new approach for congestion-control that allows TCP connections to boldly search for, and adapt to, the available bandwidth within a single RTT. Our approach relies on carefully orchestrated packet sending times and estimates the available bandwidth based on the delays experienced by these. We instantiate our new protocol, referred to as RAPID, using mechanisms that promote efficiency, queue-friendliness, and fairness. Our experimental evaluations on gigabit networks indicate that RAPID: (i) converges to an updated value of bandwidth within 1-4 RTTs; (ii) helps maintain fairly small queues; (iii) has negligible impact on regular TCP traffic; and (iv) exhibits excellent intra-protocol fairness among co-existing RAPID transfers. The rate-based design allows RAPID to be truly RTT-fair.
\end{abstract}

\section{INTRODUCTION}

"Congestion Control" can be easily listed among the top10 networking problems of the past two decades. And indeed, why not? A congestion-control protocol has no simple taskit has to adaptively discover the end-to-end spare bandwidth available to a transfer in a quick and non-intrusive manner. Simultaneously achieving these properties turns out to be a significant challenge, especially for an end-to-end protocol that receives no explicit feedback from routers/switches. Indeed, the dominant end-to-end transport protocol, TCP NewReno [1], has been shown to be abysmally slow in discovering the spare bandwidth, especially in high-speed networks and on paths that experience dynamic bandwidth.

Several alternate protocols have been proposed to address this limitation. However, as discussed in Section II, most of these protocols struggle to remain non-intrusive to other network traffic while achieving speed-consequently, these designs are still quite sluggish in probing for spare bandwidth. In particular, we show that even the so-called "high-speed" protocols may take hundreds-to-thousands of round-trip times (RTTs) to converge to a stable sending rate in gigabit networks.

In this paper, inspired by recent advances in the field of bandwidth estimation, we propose the idea that the sluggishness of transport protocols can be eliminated, without overloading the network, if we limit the impact of probing for spare bandwidth. In particular, we rely on carefully orchestrated packet sending times and use the relative delays experienced by the packets, to probe for an exponentially-wide range of rates within a single RTT - the impact of probing is limited by ensuring that the average sending rate is not high. We use this idea to design a novel approach, referred to as RAPID Congestion Control (RAPID), that exhibits three

\begin{tabular}{|c|c|}
\hline avail-bw, $\mathrm{AB}$ & available bandwidth \\
ABest & the $\mathrm{AB}$ estimate returned by the receiver \\
p-stream & multi-rate probe stream \\
$N$ & the number of packets in a p-stream \\
$P$ & packet size \\
$r_{\text {avg }}$ & the average sending rate of a p-stream \\
$r_{i}$ & the sending rate of the $(i+1)^{t h}$ packet in a p-stream \\
$m$ & the ratio of $\frac{r_{i+1}}{r_{i}}$ for all $i \in[1, N-1]$ \\
$\tau$ & the duration over which an increase in $\mathrm{AB}$ is adopted \\
\hline
\end{tabular}

TABLE I

NotATiONS USED IN THE PAPER

significantly desirable characteristics. Most notably, it reduces the time it takes a transport protocol to acquire freshlyavailable bandwidth by more than an order of magnitude. Equally significantly, by relying on a delay-based congestiondetection strategy, the protocol ensures (i) that it is friendly to transfers that use regular loss-based TCP, and (ii) that packet queues at bottleneck links are small and transient. Finally, due to its speed, RAPID also exhibits excellent intra-protocol fairness properties at small-to-medium timescales, even in network environments with heterogeneous RTTs.

Table I summarizes several notations used throughout the paper-we use the terms "packets" and "segments" interchangeably. In the rest of this paper, we describe the sluggishness of existing protocols and present our key insight in Section II. We describe the RAPID protocol mechanisms in Section III and present performance evaluation results in Section IV. We conclude in Section V.

\section{Problem Formulation}

\section{A. The Problem: Long Feedback Loop}

To understand the sluggishness issue, consider an end-toend congestion-control protocol in steady-state- the protocol continuously operates a 2-step AB-search cycle: in the searchstep, it successively probes for (by sending at) larger data sending rates. For each rate probed at, it examines performance feedback such as packet loss and high end-to-end delays that arrives after an RTT-worth of delay-this helps it estimate when the most recent sending rate was higher than the availbw. ${ }^{1}$ When a higher rate is reached, the reduction-step of the protocol reduces the sending rate to a lower value and switches back to the search-step. The speed with which a single loop of this 2-step cycle is executed fundamentally determines how quickly a protocol can acquire and adapt to changes in the avail-bw.

Note that the search-step can not be executed at a timescale smaller than an RTT-performance feedback can arrive no earlier than this time. Unfortunately, most existing protocols

\footnotetext{
${ }^{1}$ Different protocols differ in how the successive probing rates relate to the current rate, as well as in the performance measures they use as feedback. Table II summarizes these differences for some prominent protocols.
} 


\begin{tabular}{|c|c|c|c|c|c|}
\hline \multirow{2}{*}{ Protocol } & \multirow{2}{*}{$\begin{array}{c}\text { Search-step } \\
\text { (per-RTT increase in probe-rate) }\end{array}$} & \multirow{2}{*}{ Feedback-metric } & \multicolumn{3}{|c|}{ Experimentally-observed time for acquiring: } \\
\hline & & & $\mathrm{AB}=1 \mathrm{Gbps}$ & $\mathrm{AB}=2.5 \mathrm{Gbps}$ & $\mathrm{AB}=8 \mathrm{Gbps}$ \\
\hline NewReno & Additive Increase & Packet Loss & $\sim 12,000$ RTTs & $\sim 29,900$ RTTs & 〜 95,580 RTTs \\
\hline HighSpeed [2] & Multiplicative Increase & Packet Loss & $\sim 250$ RTTs & $\sim 600$ RTTs & $\sim 1,610$ RTTs \\
\hline CUBIC [3], [4] & Additive/Binary-search Increase & Packet Loss & $\sim 100$ RTTs & - & $\sim 693$ RTTs \\
\hline FAST [5] & Additive Increase & Packet Delays (\& Loss) & $\sim 30$ RTTs & $\sim 42$ RTTs & $\sim 70$ RTTs \\
\hline RAPID & Exponential Increase & Delays (\& Loss) & $\sim 4$ RTTs & $\sim 4$ RTTs & $\sim 4$ RTTs \\
\hline
\end{tabular}

TABLE II

TIME TAKEN TO ACQUIRE A MULTI-GIGABIT AB BY DIFFERENT PROTOCOLS (SEE SECTION IV-A)

execute this step at timescales much larger than this. This is because, primarily driven by the goal of not overloading the network, all previously-proposed protocols adopt two limiting design features:

1) Only a single (larger) sending rate is probed for over a round-trip time.

The protocol probes for a candidate sending rate and then waits for performance feedback, which arrives after an RTT-worth of delay. A new and larger sending rate (probeRate) is then probed for only during the next RTT time interval. This is true for all previous protocols, including recent ones, such as HighSpeed TCP, FAST, Scalable, CUBIC, and PCP [2], [3], [4], [5], [6], [7], [8]. This legacy design decision is perhaps motivated by the fact that unless the single rate is deemed acceptable (not too high), other rates should not be probed for.

2) The new rate probed for (probeRate) is not significantly larger than the previous sending rate (prevRate).

This feature is adopted primarily to prevent a single transfer from overloading the network, in case the previous rate was quite close to the avail-bw. Existing protocols differ in how the new probing rate relates to the previous rate-for most protocols, the ratio of probeRate/prevRate is only slightly larger than 1 in high-speed networks. For instance, these two quantities are additively related in NewReno and FAST, as in: probeRate $=$ prevRate $+\alpha^{*}$ MSS/RTT, where MSS is the maximum segment size allowed on the path, and $\alpha$ is set by default to 1 and 200 in the two protocols, respectively. Scalable, HighSpeed, and CUBIC rely on a multiplicative relation as in: probeRate $=\gamma^{*}$ prevRate; however, $\gamma$ is again restricted to small values. ${ }^{2}$

In addition to these two limitations, protocols that rely only on packet losses as an indicator of congestion suffer from yet another problem - these protocols may have to reach a sending rate much higher than the avail-bw (and oscillate several times around it) before stabilizing to it. This is because a loss-based protocol would need to fill up buffers in the bottleneck routers and suffer a loss before it can detect that it has acquired (and surpassed) the avail-bw.

As a result of these design limitations, most protocols-even the recent ones designed for high-speed networks [2], [7], [5], [8], [3], [4] — take a fairly long time for converging to the availbw. Table II lists, for different protocols, the experimentally-

\footnotetext{
${ }^{2} \mathrm{BIC}[4]$ relies on a combination of additive-increase and a binary search based method after the $\mathrm{AB}$ is discovered for the first time-here, the ratio probeRate/prevRate depends on the past probing history.
}

observed times taken by a single transfer for acquiring a spare capacity of $1 \mathrm{Gbps}$ or higher (say, after experiencing a packet loss). ${ }^{3}$ We find that even the fastest of high-speed protocols can take hundreds of RTTs for converging to the avail-bw.

\section{B. Key Insight: Limit Probing Volume}

We believe that all of the above design limitations can be done away with, without overloading a network or inducing buffer overflow, if one limits the volume (and impact) of probing for a larger sending rate. In fact, we claim that a protocol can boldly probe for an exponentially-wide range of candidate sending rates within a single RTT, if it: (i) uses carefully-orchestrated inter-packet gaps, and (ii) relies on relative packet delays for estimating avail-bw. Any associated overloading impact can be avoided by using the following guidelines:

1) Achieve a rapid $A B$ search: Probe for an exponentiallywide range of candidate sending rates within a single RTT. However, send extremely small probes (of one packet each) at each candidate rate in order to limit to very small timescales the overloading impact of the large rates.

2) Avoid persistently overloading the network path: Ensure that the average rate of packet transmission does not exceed the most-recently discovered estimate of availbw. This implies that some of the rates in the abovesuggested exponential range will be smaller than this estimate, and some will be larger.

We use these ideas to design a new protocol, referred to as RAPID Congestion Control (RAPID), and show that it can adapt to fairly large changes in AB within 1-4 RTTs. Furthermore, RAPID can avoid persistently large router queues due to its primary focus on avoiding network overload. The benefits of the protocol are especially significant in dynamic bandwidth environments and high-speed networks.

It is important to note that several protocols such as Vegas, FAST, and PCP also rely on packet delays (instead of packet losses) for detecting congestion; however, none of these protocols exploit inter-packet gaps to probe for a wide range of rates within a single RTT. ${ }^{4}$

We next present the basic mechanisms used in RAPID.

\footnotetext{
${ }^{3}$ These experiments were run on the ns- 2 simulator, and a packet size of 1040 B was used-see Section IV-A for details. The ns-2 implementation of CUBIC failed to run at $2.5 \mathrm{Gbps}$.

${ }^{4}$ The rate-based PCP protocol [6] also adopts the idea of "limiting the probe volume". However, it probes for only a single larger rate per RTT and has an $\mathrm{AB}$-search speed similar in magnitude to that of existing protocols. In all fairness, the protocol primarily focusses on minimizing response times in under-utilized networks - it has not even been evaluated for large transfers in high-speed networks.
} 


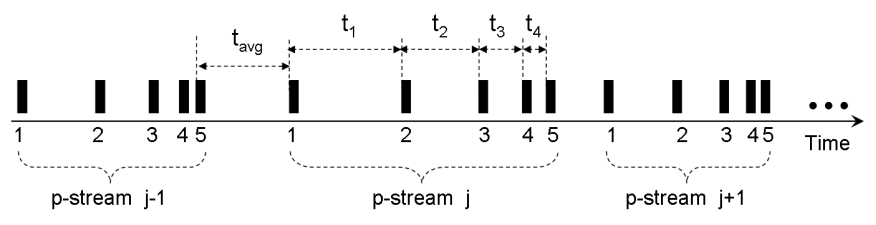

Fig. 1. Illustration of a p-stream (here, $t_{i}=\frac{P}{r_{i}}$ and $t_{a v g}=\frac{P}{r_{a v g}}$ )

\section{RAPID CONGESTION CONTROL}

Unlike many congestion-control protocols, RAPID employs a multi-rate based transmission policy and relies on relative packet delays for estimating avail-bw. While the RAPID design is motivated by the primary goal of shrinking the timescales at which congestion-control operates, several equally-important goals are given due consideration in the design process [9]. Most significantly, a RAPID network strives to (i) maintain a low buffer occupancy at congested router links, (ii) achieve a fair sending rate when several RAPID transfers co-exist, and (iii) remain friendly to regular low-speed TCP transfers. Below, we describe the basic mechanisms used for achieving each of these.

\section{A. Acquiring $A B$ Within a Few RTTs}

1) Rate-based packet transmission at the sender: When there is sufficient data to send, a RAPID sender continuously transmits data in logical groups of $N$ packets each, referred to as a multi-rate probe stream ( $p$-stream). ${ }^{5}$ The $i$-th packet in a p-stream is sent at a rate of $r_{i-1}$; this also implies that the sending times of packets $i$ and $i-1$ differ by $\frac{P}{r_{i-1}}$, where $P$ is the size of packet $i$ (see Figure 1). The sender explicitly controls/manages the average sending rate of a pstream, referred to as $r_{\text {avg }}$, which is given by:

$$
r_{a v g}=\frac{N-1}{\frac{1}{r_{1}}+\frac{1}{r_{2}}+\ldots+\frac{1}{r_{N-1}}}
$$

Further, for all $i>1, r_{i}>r_{i-1}{ }^{6}$

2) AB-estimation analysis at the receiver: We observe the inter-packet gaps in a p-stream at the receiver, and use these for estimating avail-bw in the same manner as the PathChirp bandwidth estimation tool [10]. Recent evaluations have shown that PathChirp estimates avail-bw with good accuracy in multihop settings, while incurring the least overhead among existing tools [11].

Like PathChirp, when a RAPID receiver receives all packets of a p-stream, it computes the $\mathrm{AB}$ by looking for increasing trends in the intended inter-packet spacings. This analysis relies on the concept of self-induced congestion. Intuitively, if $q_{i}$ is the queuing delay experienced at a bottleneck link by

\footnotetext{
${ }^{5}$ Unlike window-based protocols, the sender does not stall, waiting for acknowledgments. Also, if data for only $k<N$ packets is available, a pstream of $k$ packets is sent instead. Note that if $k \leq 2$, the sender sends these packets at a uniform rate of $r_{\text {avg }}$.

${ }^{6}$ The gap between the first packet of a p-stream and the last packet of the previous p-stream is set to $r_{\text {avg }}$. This can also be stated as: $r_{0}=r_{\text {avg }}$. Typically, $r_{0}>r_{1}$.
}

the $i$-th packet in a p-stream, then:

$$
\begin{array}{ll}
q_{k}=0, & \text { if } r_{k} \leq A B \\
q_{k}>q_{k-1}, & \text { otherwise }
\end{array}
$$

Thus, if $i^{*}$ is the first packet in a p-stream such that $r_{i^{*}-1} \geq$ $A B$, then each of the packets $\left[i^{*}, \ldots, N\right]$ will queue up behind its previous packet at the bottleneck link (since $r_{i}>r_{i-1}$, for all $i>1$ )—due to this "self-congestion", each of these packets will experience a larger one-way delay (and a larger increase in the pre-set inter-packet gap) than its predecessor. Thus, the smallest rate $r_{i^{*}-1}$ at which the receiver observes an increasing trend in the inter-packet gaps can be used to compute an estimate of the current avail-bw as: ABest $=$ $r_{i^{*}-1} \cdot{ }^{7}$ The actual analysis uses several heuristics to account for bursty cross-traffic - we refer the reader to [10] for details and the precise formulation.

The receiver encodes the value of ABest obtained from the most recent $\mathrm{p}$-stream in the acknowledgments sent to the sender.

3) Transmitting in a non-overloading, responsive manner: When the sender receives an $A B e s t$ value, it updates the $r_{\text {avg }}$ of the next p-stream as: $r_{a v g}=A B e s t$. Thus, the transfer acquires an average sending rate equal to the estimated availbw within an RTT. The sender then selects an appropriate set of rates, $r_{1}, \ldots, r_{N-1}$, for the next p-stream such that the average of these is equal to $r_{\text {avg }}$, as computed in Eqn (1).

The above mechanism helps simultaneously achieve two desirable properties. First, by setting $r_{a v g}$ equal to the estimated avail-bw, RAPID helps to ensure that the average load on the bottleneck link does not exceed its capacity-this is crucial for maintaining small and transient queues at the bottleneck links. Second, by selecting a set of rates which includes values larger $\left(r_{N-1}\right)$ as well as smaller $\left(r_{1}\right)$ than $r_{\text {avg }}$, a p-stream is able to simultaneously probe for both increase and decrease in the current end-to-end avail-bw - this greatly helps the RAPID sender in quickly detecting and adapting to changes in AB.

4) Setting $\left[r_{1}, \ldots, r_{N-1}\right]$ (speeding up the search process): Each p-stream probes for the range of sending rates given by: $\left[r_{1}, \ldots, r_{N-1}\right]$. Note that for a given $r_{a v g}$ and $N$, there are infinite choices for this set of rates, such that they satisfy Eqn (1). However, the larger is the ratio $\frac{r_{N-1}}{r_{1}}$, the faster would be the AB-search process - this is because a single p-stream now probes for a wider range of rates.

So for instance, while these rates could be additively-related, a faster search will be obtained by using a multiplicativerelation as in:

$$
r_{i}=m^{i-1} * r_{1}, \quad 1<i<N
$$

RAPID adopts the above relation. Given $r_{\text {avg }}$, Eqns (1) and (4) can be used to compute $r_{1}$ as:

$$
r_{1}=\frac{m^{N-1}-1}{(N-1)(m-1) m^{N-2}} r_{a v g}
$$

\footnotetext{
${ }^{7}$ If no increasing trend is detected in a p-stream, $r_{N-1}$ is taken as the $\mathrm{AB}$ estimate. Also, if the increasing trends starts at the first or second packet $\left(i^{*} \leq 2\right), \frac{r_{1}}{2}$ is returned as the $\mathrm{AB}$ estimate.
} 


\begin{tabular}{||c||c|c||}
\hline \hline \# of RTTs & $r_{a v g}$ & Rates that can be probed for \\
\hline \hline RTT 0 & $x_{0}$ & $0.45 x_{0}-3.22 x_{0}$ \\
\hline RTT 1 & $3.22 x_{0}$ & $1.5 x_{0}-10.7 x_{0}$ \\
\hline RTT 2 & $10.7 x_{0}$ & $4.7 x_{0}-33.4 x_{0}$ \\
\hline RTT 3 & $33.4 x_{0}$ & $15 x_{0}-108 x_{0}$ \\
\hline RTT 4 & $108 x_{0}$ & $48 x_{0}-346 x_{0}$ \\
\hline RTT 5 & $346 x_{0}$ & $156 x_{0}-1115 x_{0}$ \\
\hline \hline
\end{tabular}

TABLE III

AB-ACQUIRING SPEED BY A RAPID SENDER

$r_{2}, \ldots, r_{N-1}$ can then be computed from Eqns (4) and (5).

a) Selecting $m$ and $N$ : For a given $N$, two conflicting considerations guide the choice of $m$. A larger value of $m$ would also result in a larger ratio of $\frac{r_{N-1}}{r_{1}}$, and would improve the speed as well as adaptivity of the AB-search process. A smaller $m$, on the other hand, would result in a smaller ratio of $\frac{r_{i+1}}{r_{i}}$-this would result in a finer rate granularity with which the avail-bw is probed. A coarse-granularity estimate of avail-bw would prevent a collection of RAPID senders from efficiently utilizing the bottleneck link.

The selection of $N$ is also faced by two opposing considerations. For a given $m$, a larger value of $N$ would improve the $\mathrm{AB}$-search range $\left(\frac{r_{N-1}}{r_{1}}\right)$. However, a larger p-stream would also be more intrusive to cross-traffic (more packets would be sent at a rate larger than $\left.r_{a v g}\right)$ at bottleneck links.

RAPID adopts the default values of $N=30$ and $m=$ 1.07 (7\% granularity in rates probed for). These values have been selected after controlled experimentation under diverse topology and traffic settings - we do not present this sensitivity analysis here due to space constraints. Details can be found in [12].

The above choices of $m$ and $N$ yield: $r_{1} \approx 0.45 * r_{\text {avg }}$ and $r_{N-1} \approx 3.22 * r_{\text {avg }}$. This enables a RAPID sender to probe for freshly-available spare bandwidth spanning several orders of magnitude within a few RTTs (see Table III).

5) Achieving a Quick-yet-Slow Start: RAPID faces a similar dilemma as all congestion-control protocols-how to obtain the initial $A B e s t$ (or the initial $r_{a v g}$ ) for a new transfer? The main concern here is that the initial choice of $r_{\text {avg }}$ may be too high for a given network path. We address this issue by being only as aggressive as the TCP Slow-Start mechanism (which is also adopted by most other protocols). Specifically, in slow-start, a RAPID sender sends only as many packets in an RTT as would a TCP transfer-fortunately, the ability of a p-stream to probe for multiple rates within an RTT makes the RAPID slow-start terminate much earlier than other protocols.

In the slow-start phase, a RAPID sender sends only a single p-stream over an RTT. Further, we initialize $N=2$, and double the value of $N$ over successive RTTs, up to a maximum value of $16 .^{8}$ For constructing the p-streams, we use a multiplicative factor of $m=2$ throughout slow-start-thus, the granularity with which a RAPID sender probes for availbw during slow-start is coarse, but is the same as all existing

\footnotetext{
${ }^{8}$ Note that this process is no more aggressive than the slow-start adopted by most protocols, which multiplicatively increases the number of packets sent over an RTT in exactly the same manner (and start with an initial value of 1 or 2 segments). Also note that the slow-start threshold is usually set to a much higher value than 16 segments.
}

\begin{tabular}{||c||c|c||}
\hline \hline \# of RTTs & $N$ & Range of rates probed for \\
\hline \hline RTT 1 & 2 & $0-100 \mathrm{Kbps}$ \\
\hline RTT 2 & 4 & $100-800 \mathrm{Kbps}$ \\
\hline RTT 3 & 8 & $800 \mathrm{Kbps}-102 \mathrm{Mbps}$ \\
\hline RTT 4 & 16 & $102 \mathrm{Mbps}-3342 \mathrm{Gbps}$ \\
\hline \hline
\end{tabular}

SPEED OF RAPID SLOW-START

protocols (that double their window size every RTT) [1], [13]. Unlike most protocols, however, the AB-search speed is quite high-since it relies on an AB-estimation analysis, a RAPID transfer in slow-start discovers avail-bw much earlier than any other protocol.

For a new RAPID transfer, we initialize $r_{a v g}$ to a small value (100Kbps) - this implies that, in the first RTT, the transfer sends $N=2$ packets at a rate of $r_{1}=100 \mathrm{Kbps}$. If the receiver returns $A B e s t<r_{N-1}$, we exit slow-start; if not (which implies, $A B e s t=r_{N-1}$ ), we double the value of $N$, set $r_{1}=A B e s t$, and send the next p-stream. ${ }^{9}$ This process is repeated till the receiver returns an $A B$ est value less than $r_{N-1}$. Following this, we switch to the steady-state RAPID mode, in which $m=1.07$ and $N=30$.

Table IV illustrates the number of RTTs that a single RAPID transfer would take to probe for different amounts of avail-bw in slow-start-an RAPID sender can probe for more than 1 Tbps in just 4 RTTs, while being no more aggressive than TCP slow-start! In Section IV, we show that existing protocols take much longer.

6) Dealing With Packet Losses: Like in NewReno, packet losses are recovered using the Retransmission Timeout and Fast Retransmit/Recovery mechanisms. After loss recovery, the sender reduces $r_{a v g}$ by a multiple of 0.5 and resumes sending of p-streams.

\section{B. Achieving Fairness Among Co-existing RAPID Transfers}

The RAPID design should justifiably raise fairness concerns when multiple RAPID transfers share a bottleneck link: how do the p-streams of different transfers interact-do the transfers obtain a fair share of the avail-bw? In this section, we address two sources of unfairness that have been identified in the literature [14], [15].

1) RTT-unfairness: Most window-based congestion control protocols have been shown, both experimentally and analytically, to suffer from RTT-unfairness - transfers with a long RTT get a lower throughput than short-RTT transfers [14], [15], [5]. This happens because window-based protocols update their sending rates once per RTT-in heterogeneous RTT environments, this RTT-dependence results in differences in the rate updating frequency as well as rate increments, and results in a bias against long RTT transfers [15].

Fortunately, the rate-based design of RAPID is not influenced by the value of RTT-a RAPID sender continuously send p-streams, for both long and short RTT transfers. The rate-updating frequency (once per p-stream) as well as rate increment (determined by ABest) are independent of the RTT - consequently, and by design, RAPID truly does not

\footnotetext{
${ }^{9}$ Note that in slow-start, RAPID sets $r_{1}$ (and not $r_{a v g}$ ) equal to ABest.
} 
suffer from RTT-unfairness. Our experiments in Section IV-B confirm this.

2) Bias due to rate-proportional feedback frequency: As described so far, however, the RAPID design is likely to be unfair for a different reason. The rate at which a RAPID sender receives ABest values (once per p-stream), is directly proportional to the current value of $r_{a v g}$ and is given by: $\frac{r_{a v g}}{N * P}$, where $P$ is the packet size. Consequently, a RAPID transfer that has achieved a larger sending rate will receive more frequent notifications of any spare capacity that becomes available, than a co-existing transfer with a lower $r_{a v g}$ thus, the former is likely to attain an even higher sending rate than the latter. In fact, such differences in the rate at which feedback arrives have been shown to result in unfair throughput allocations even in window-based protocols [15].

To ensure that co-existing RAPID transfers achieve a fairshare of the avail-bw, we explicitly equalize the rate at which RAPID senders converge to the ABest values they receive. For this, we select a parameter $\tau$ that represents the common (large) time interval over which any RAPID sender, independent of its sending rate, would converge to an increase in avail-bw. Specifically, when a sender receives an ABest that is larger than its current $r_{\text {avg }}$, it updates $r_{\text {avg }}$ as:

$$
r_{a v g}=r_{a v g}+\frac{l}{\tau}\left(\text { ABest }-r_{a v g}\right)
$$

where $l$ is the duration of the most-recent p-stream, which is given by: $l=\frac{N * P}{r_{\text {avg }}}$. The above filter is used to repeatedly update $r_{\text {avg }}$ for all subsequent p-streams, till $r_{\text {avg }}$ converges to ABest. The effect of this filtering mechanism is that it would take a RAPID sender roughly $\tau$ time units (or $\frac{\tau}{l}$ p-streams) for converging to an updated value of ABest-for a transfer with a small value of $r_{\text {avg }}, \frac{l}{\tau}$ will be close to 1 and the sender will immediately adopt the $A B e s t$ as its new $r_{\text {avg }}$. Thus, even though the feedback frequency depends on the sending rate of a RAPID transfer, the rate-increment frequency does not. ${ }^{10}$ Our experiments in Section IV-B show that this mechanism helps achieve excellent fairness among RAPID transfers. $\tau$ is set by default to $200 \mathrm{~ms}$.

\section{Remaining TCP-Friendly}

RAPID, like FAST, is quite non-intrusive to regular lowspeed TCP NewReno transfers. The prime reason for this is that these protocols rely on increased packet delays for detecting network congestion, whereas TCP reduces its sending rate only on witnessing packet losses. When a router carrying both TCP and RAPID transfers gets congested, the RAPID transfers would respond to the congestion (and reduce their sending rates) much earlier than the TCP transfers would. This would ensure that the performance of the low-speed TCP transfers is not significantly impacted due to the presence of high-speed RAPID transfers.

We next experimentally evaluate how well the above mechanisms achieve the stated goals for RAPID.

\footnotetext{
${ }^{10}$ For stability reasons, we do not use the above filter when the avail-bw decreases—details are provided in [12].
}

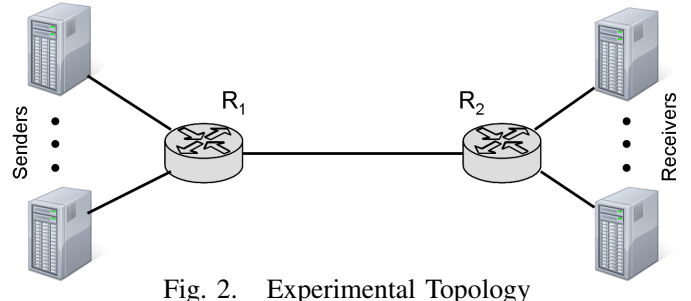

Fig. 2. Experimental Topology

\section{EXPERIMENTAL EVALUATION}

There are at least three types of concerns that the RAPID design is likely to raise in the minds of a reader: does a short p-stream really help RAPID in accurately estimating avail-bw, especially in high-speed networks? RAPID seems aggressive in the rates it probes for-is it really friendly to router queues and competing low-speed TCP traffic? And, how do the AB-estimation processes of co-existing RAPID transfers interact-don't they interfere with each other (and do they get a fair share of the avail-bw)? In this section, we address these concerns by experimentally evaluating RAPID. We have also experimentally studied: (i) sensitivity of RAPID to parameter settings, (ii) fairness among multiple high-speed protocols, and (iii) RAPID performance in multi-hop settings [12]; for space constraints, however, these are omitted from this paper.

We use the ns-2 simulator [16] for our evaluations. We have implemented RAPID in ns-2.33 and have re-used the NewReno code base for dealing with loss detection and recovery. We also use publicly-available ns- 2 implementations of three other protocols for comparison, namely HighSpeed TCP, CUBIC, and FAST TCP [17] - the first two are loss-based protocols with fairly different window-growth functions; FAST is a high-speed version of the delay-based Vegas protocol. ${ }^{11}$

For several of our simulations, we rely on a simple dumbbell topology in which multiple sources are aggregated at a single bottleneck link $\left(R_{1}-R_{2}\right.$ in Fig 2$)$-this bottleneck link is the only link shared by co-existing transfers. All links other than the bottleneck link have a transmission capacity of $10 \mathrm{Gbps}-$ the bottleneck link capacity is set by default to $1 \mathrm{Gbps}$, but is reduced for some experiments. All links are provisioned with a delay-bandwidth product (DBP) worth of buffers, where the delay is the average end-to-end propagation delay for transfers (specified for each experiment), and the bandwidth is that of the bottleneck link (also specified). We set the maximum size of each network-layer packet to $1040 \mathrm{~B}$.

The main performance statistics we are interested in are throughput obtained by transfers instantiated between the sender and receiver nodes as well as the queue build-up on the bottleneck link. We sample each of these statistics periodically at regular intervals of $50 \mathrm{~ms}$ each. In what follows, we summarize our experiments and observations.

\footnotetext{
${ }^{11}$ We rely on the default parameter settings configuration for all protocols, other than for FAST. FAST exhibited severe oscillations for the experiments of Section IV-A when run with default ns-2 parameters-for the evaluations presented here, we have instead relied on bottleneck capacity-specific parameters used in sample test scripts supplied along with the implementation [17] (experiments with other parameter settings are included in [12]).
} 


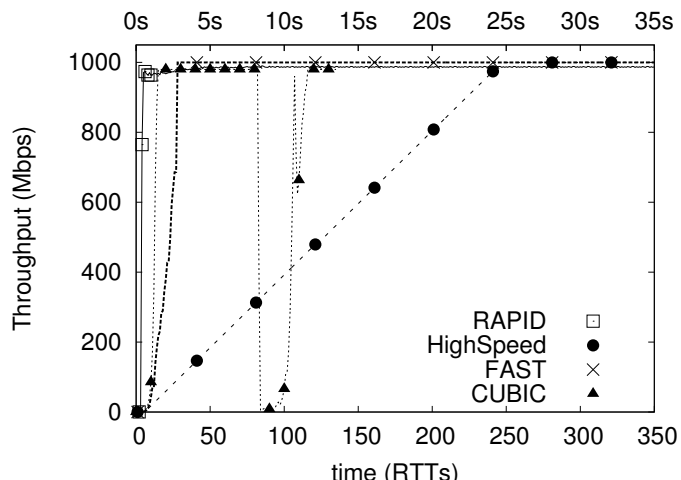

(a) Throughput vs. Time

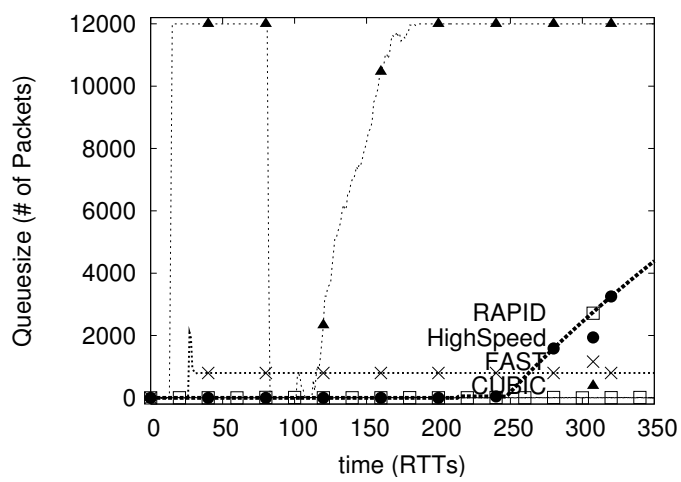

(b) Bottleneck Queue vs. Time

Fig. 3. Performance of Slow Start on a 1 Gbps Network

\section{A. Speed of Acquiring Spare Bandwidth}

There are at least two types of scenarios in a high-speed network where the ability of a congestion-control protocol to acquire spare bandwidth quickly is crucial. The first is during slow-start, when a transfer begins without any advance knowledge of the avail-bw, and the second is when the availbw suddenly changes by a large amount (perhaps due to the arrival of additional traffic). In our first set of experiments, we compare the performance of RAPID with that of other protocols by simulating examples of both of the above scenarios. For all of the experiments in this section, we simulate a 1 Gbps bottleneck link $\left(R_{1}-R_{2}\right.$ in Fig 2$)$ and set the end-toend propagation delay to $100 \mathrm{~ms}$.

1) Slow-start in High-speed Networks: In the first set of experiments, we simulate a single transfer from a sender node to a receiver node (see Fig 2). Each experiment uses a different underlying congestion-control protocol. Fig 3(a) plots as a function of time, the throughput obtained by the transfer with different protocols. Fig 3(b) plots the queue buildup observed over time at the bottleneck router. We find that:

- HighSpeed TCP takes the longest time (250 RTTs) for acquiring a sending rate of 1 Gbps. And once it acquires that sending rate, being a loss-based protocol, it starts filling up the bottleneck queues. ${ }^{12}$

CUBIC is faster, initially acquiring 1 Gbps after merely 10 RTTs-but it also fills up the bottleneck buffers as

\footnotetext{
${ }^{12}$ We also ran this experiment with the NewReno protocol, which took around 12000 RTTs to acquire the 1 Gbps avail-bw.
}

quickly, later leading to packet losses and a drop in throughput. It eventually stabilizes to 1 Gbps after 110 RTTs. Being a loss-based protocol, it also maintains nearly full buffers at the bottleneck link.

- FAST TCP is less aggressive in filling up router queues and is able to acquire avail-bw faster-within 30 RTTs.

- RAPID is significantly faster than all other protocols, acquiring the 1 Gbps bandwidth in just 4 RTTs-this is exactly as was predicted by the design in Section III-A5. Furthermore, the single RAPID transfer induces very low queuing (less than 20 packets) on the bottleneck router.

Table II summarizes the slow-start delays of these protocols for bottleneck link $\left(R_{1}-R_{2}\right)$ capacities of $1 \mathrm{Gbps}, 2.5 \mathrm{Gbps}$, and $8 \mathrm{Gbps}$ - the performance of most protocols worsens at higher speeds, while RAPID scales as expected.

2) High-speed Networks with Dynamic AB: In the second set of experiments, we simulate a network for 500 seconds, and introduce 4 constant-bit-rate (cbr) traffic streams on the bottleneck link according to the following schedule: $c b r$ 1 exists from 50-400 seconds, $c b r-2$ exists from 100-150 seconds, $c b r-3$ exists from 250-350 seconds, and $c b r-4$ exists for a small duration from 460-462 seconds. Each cbr stream has a bit-rate of 200 Mbps. The spare bandwidth left on the network is plotted in the top-row plots of Figs 4(a)-(d) using a faint dotted line.

We use this setup to run a set of experiments in which we introduce a single long-lived transfer at time 1 second, and respectively, run it over CUBIC, HighSpeed, FAST, and RAPID. The throughput obtained by the transfers and the router queue sizes are plotted, respectively, in the top and bottom rows of Fig 4. We find that:

1) HighSpeed and CUBIC, which are both loss-based protocols, experience heavy packet losses when the avail-bw reduces suddenly (and even when it does not change). This is because a loss-based protocol induces persistent queuing in the bottleneck buffers-any sudden decrease in $A B$ overflows the buffers causing multiple packet losses. When this happens, the throughput of the transfer drops significantly and each protocol then takes tens-tohundreds of RTTs for regaining throughput.

HighSpeed and CUBIC are also slow in acquiring spare bandwidth when the avail-bw increase suddenly (for example, at 150s, 250s, and 350s) - this is simply due to the slow AB-search speed of these protocols. Note that if these protocols already have a large number of packets in the router buffers, they may be able to utilize spare bandwidth immediately-loss-based protocols are known to trade-off low latency for high throughput performance.

2) Delay-based FAST maintains smaller router queues in steady-state. However, when the avail-bw decreases suddenly, FAST is unable to react quickly and causes buffer overflow.

3) The RAPID transfer maintains very low queuing and is able to avoid packet losses, even when the avail- 

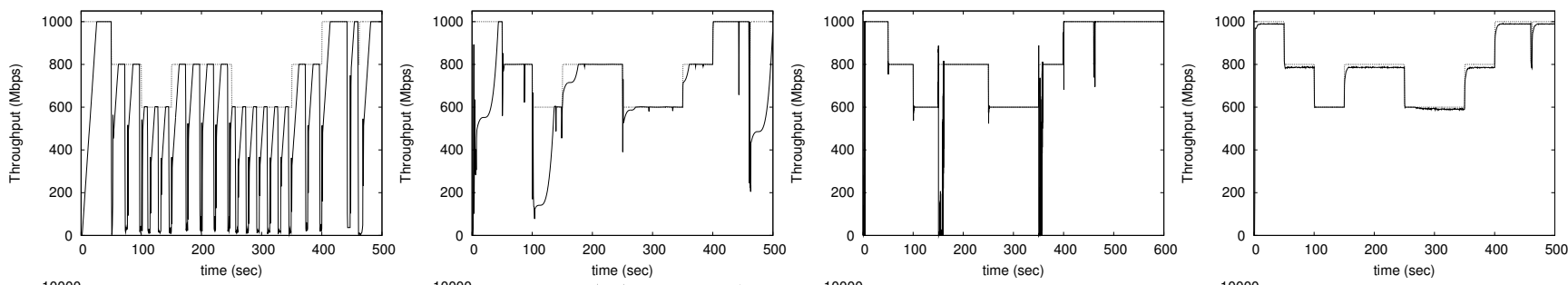

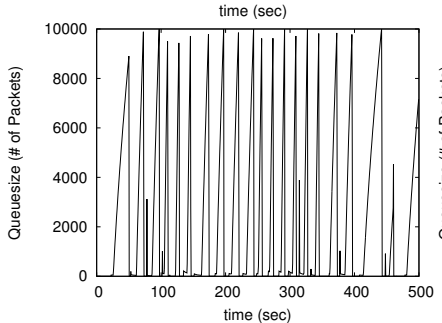

(a) HighSpeed

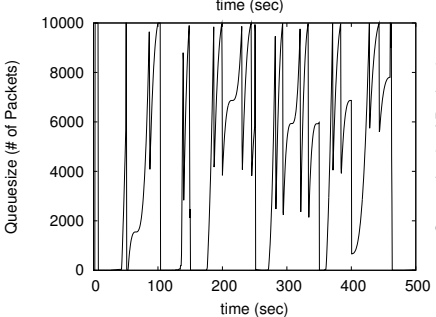

(b) CUBIC

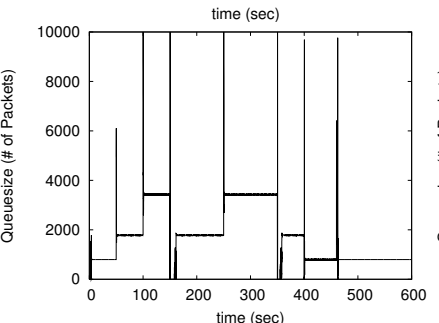

(c) FAST

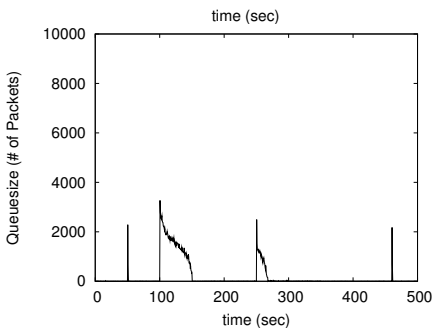

(d) RAPID

Fig. 4. Performance in a Dynamic Bandwidth 1 Gbps Network

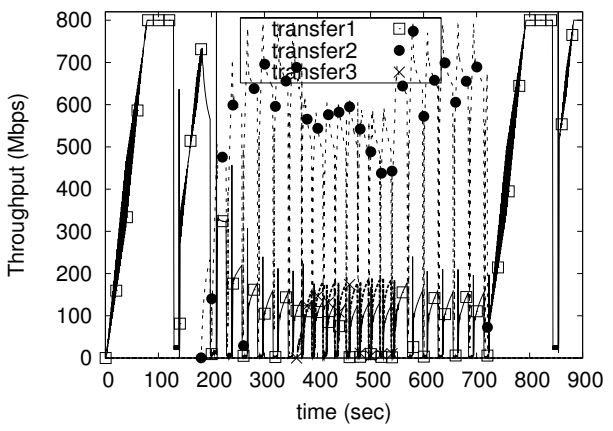

(a) HighSpeed

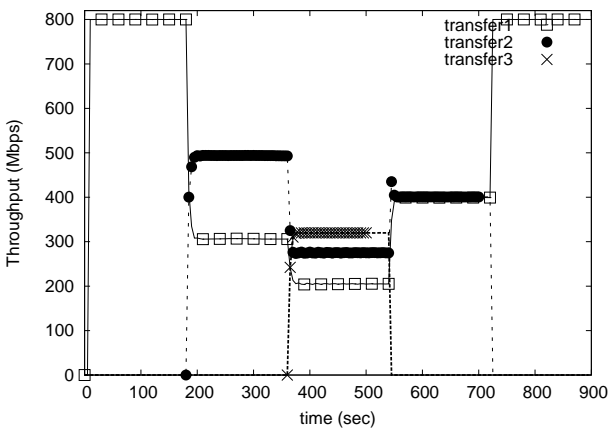

(b) FAST

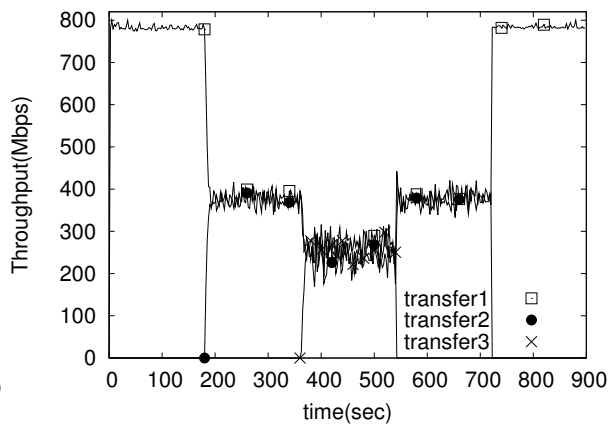

(c) RAPID

Fig. 5. Intra-protocol Fairness

bw decreases suddenly. More importantly, though, the protocol is able to very quickly acquire additional spare bandwidth that becomes available — and it does so without maintaining large queues in routers!

\section{B. Intra-protocol Fairness}

We next evaluate fairness when multiple RAPID transfers share the bottleneck link. Our objective is to specifically evaluate fairness in heterogeneous RTT environments.

1) Dynamics Among Co-existing Transfers: Our first set of experiments is inspired by a similar experiment presented in [5], which evaluates the ability of a new transfer to acquire fair share of bandwidth from a pre-existing transfer-in that paper, FAST was shown to be better than other protocols in achieving fairness (when compared against HighSpeed as well as BIC). A bottleneck capacity of $800 \mathrm{Mbps}$ was used in that experiment-we use the same by setting the transmission capacity of $R_{1}-R_{2}$ in Fig 2 to $800 \mathrm{Mbps}$.

For the experiment, we simulate three transfers- the first lasts from 0-900s and has an RTT of $200 \mathrm{~ms}$, the second lasts from 180-720s and has an RTT of $100 \mathrm{~ms}$, and the third lasts from 360-540s and has an RTT of $150 \mathrm{~ms}$. Fig 5 plots the throughput obtained by the three transfers with different underlying protocols.

We find that both HighSpeed and CUBIC (latter not plotted here to improve visualization of Fig 5) can be quite unfair in the throughput allocated among the three transfers- the second transfer (which has the smallest RTT) dominates over the other two transfers. Although FAST significantly improves upon this behavior, it still exhibits unfairness. This has also been observed in [5].

Fig 5(c) shows that RAPID allocates similar throughput to all transfers, irrespective of their RTTs-RAPID thus does not suffer from RTT-unfairness. Furthermore, the second transfer is able to acquire a fair share even though the pre-existing first transfer had a high throughput of $1 \mathrm{Gbps}$; this illustrates that the filter mechanism added in Section III-B successfully eliminates from RAPID any bias due to rate-proportional feedback frequency.

Fig 5(c) also illustrates that the convergence of the transfers to a fair share occurs at a fairly small timescale-we study the fairness timescale in more detail below.

2) Fairness Among Large Number of RAPID Transfers: We next evaluate how well the RAPID intra-protocol fairness scales when a larger number of connections are aggregated. For this, we conduct experiments in which a $1 \mathrm{Gbps}$ bottleneck link is shared by $n$ long-lived RAPID transfers for 600 seconds-we vary $n$ from 2 to 100 across experiments. The RTTs of the $n$ transfers are selected uniformly randomly from two ranges: $60-80 \mathrm{~ms}$ and $135-165 \mathrm{~ms}$. The start-times of the 


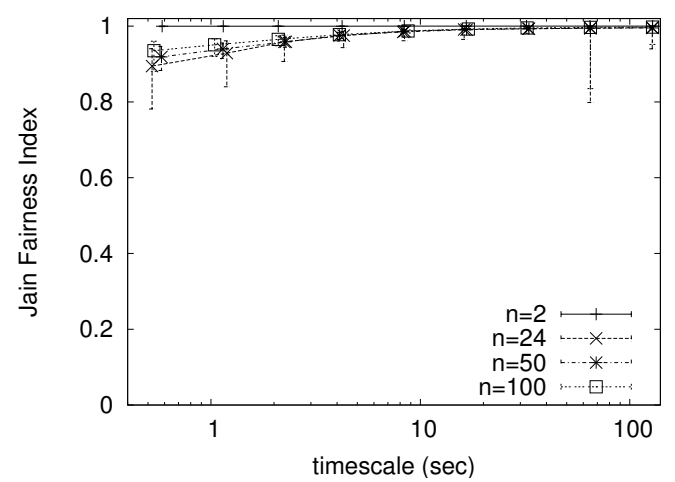

Fig. 6. Intra-protocol Fairness (Large Number of Transfers)

connections are selected uniformly randomly between $0-20 \mathrm{~s}$.

Starting at 20 seconds, the time-series of throughput obtained by each transfer are logged at several different timescales ranging from $500 \mathrm{~ms}-128 \mathrm{~s}$. For each timescale, and for each logging instance of the associated time-series, we compute the Jain Fairness Index [18] of the throughput achieved by the $n$ transfers. Fig 6 plots the median, 10th, and 90th percentiles (latter plotted as error bars) of the distribution of the fairness index, as a function of the timescale at which the throughput data is observed (for $n=2,24,50,100$ ).

We find that RAPID ensures excellent fairness (median fairness index $>0.8$ ) among a large number of co-existing transfers with heterogeneous RTTs - this is true even at timescales as small as $500 \mathrm{~ms}$. Further, even the 10 -percentile values of the indices are fairly high, indicating that it is quite rare for even transient "unfair" episodes-in which some connections obtain much less throughput than others- to occur.

\section{Co-existence with Low-speed TCP Traffic}

Finally, we evaluate the impact of high-throughput RAPID transfers on a realistic mix of regular TCP transfers that coexist on a bottleneck link. For this, we use the publiclyavailable TMIX traffic generator code for ns-2, which generates an empirically-derived TCP traffic mix that is representative of TCP traffic aggregates observed on production Internet links [19]. TMIX simulates application-level socket-writing behavior and runs over the TCP protocol-it, consequently, generates response TCP traffic. Our objectives are to study: (i) how a high-throughput transfer might impact the performance of connections in such a traffic mix, and (ii) how effectively can a high-throughput transfer utilize the avail-bw with such dynamic (and realistic) cross-traffic.

We use TMIX to generate TCP traffic at an average offered load of $70 \mathrm{Mbps}$ for 30 minutes and drive it through a bottleneck link $\left(R_{1}-R_{2}\right.$ in Fig 2$)$ of 100 Mbps - the bottleneck buffers are set to 750 packets (based on the RTT of the $\mathcal{L}$ transfer described below). For the set of TCP connections simulated in our TMIX experiment, Fig 7(a) plots the distributions of per-connection RTTs-we find that these are fairly diverse (ranging from 10ms LANs to long-distance transfers). Fig 7(b) plots the (complementary) distribution of the number of bytes transferred in each connection-the traffic mix is

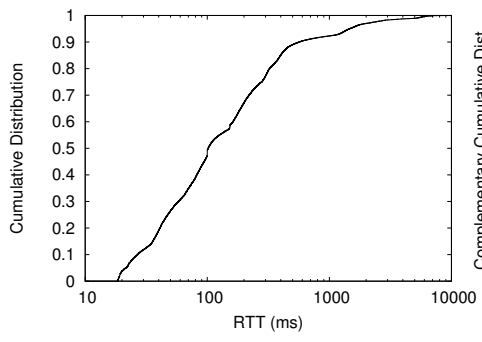

(a) Distribution of Transfer RTTs

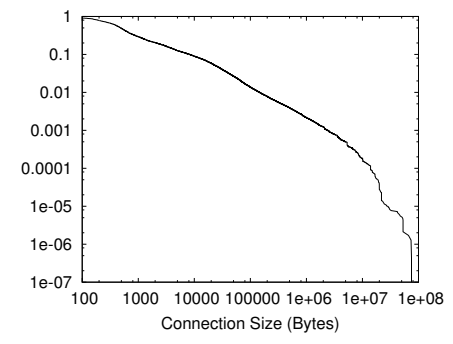

(b) Distribution of Transfer Sizes
Fig. 7. Statistics of TMIX Transfers

typical of that found on the Internet (a majority of small mice connections, but a few heavy elephants). All statistics presented below are collected from roughly the middle 20 minutes of the experiments (to exclude the ramp-up and rampdown behavior of the traffic generator).

We first simulate only the TMIX traffic and observe the aggregate throughput and queue sizes at the bottleneck router at a timescale of $1 \mathrm{~s}$ - these are plotted respectively in Fig 8(a) and $8(\mathrm{~b})$. We find that although the average offered load of the TMIX aggregate is $70 \mathrm{Mbps}$, the traffic is fairly bursty; the short-term load can vary from $50-100 \mathrm{Mbps}$. This causes the router queues to vary rapidly from $0 \sim 80$ packets (and occasionally to larger amounts). Thus, the TMIX aggregate represents bursty cross-traffic that causes the avail-bw on the bottleneck router link to vary dynamically.

We then re-run the TMIX experiment and add a single bulk transfer (henceforth, referred to as $\mathcal{L}$ ) that shares the bottleneck link and has an RTT of $60 \mathrm{~ms}$-we repeat this experiment three times with $\mathcal{L}$ running over HighSpeed, FAST, and RAPID, respectively. In each of these experiments, we also $\log$ the throughput observed by $\mathcal{L}$, the queue sizes at the bottleneck router, as well as the aggregate throughput of the bottleneck router. Figs 8(c), 8(e), and 8(g) plot the throughput of $\mathcal{L}$ observed with HighSpeed, FAST, and RAPID, respectively. Figs 8(d), 8(f), and 8(h) plot the router queue sizes, respectively. We find that:

1) Fig 8(d) shows that the loss-based HighSpeed transfer fills up (and overflows) the router queues at a frequent rate-consequently, the responsive TCP connections in the TMIX cross-traffic suffer packet losses and reduce their sending rates. The HighSpeed $\mathcal{L}$ transfer is, thus, able to obtain a throughput much higher than the spare bandwidth available in the TMIX-only experiment.

2) The $\mathcal{L}$ transfer using FAST TCP maintains an almost constant throughput of around $13 \mathrm{Mbps}$, when running along with TMIX cross-traffic. This suggests that FAST is unable to adjust to and effectively utilize the dynamically-varying avail-bw on the bottleneck link. Fig 8(f) shows that router queues in this experiment, although much smaller than with HighSpeed, are large.

3) The RAPID-based $\mathcal{L}$ transfer is able to utilize the spare bandwidth fairly effectively-the fast AB-search speed of RAPID is crucial in achieving this behavior. Fig 8(h) shows that RAPID does do while increasing the router 


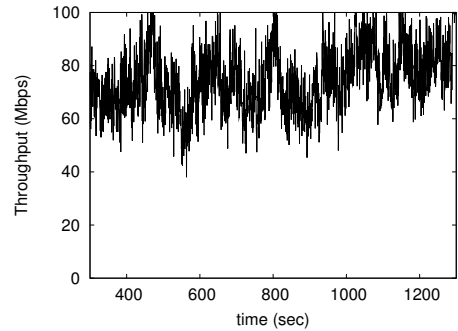

(a) Throughput (TMIX only)

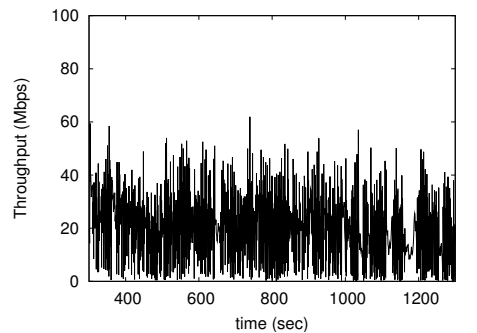

(c) Throughput (HighSpeed transfer)

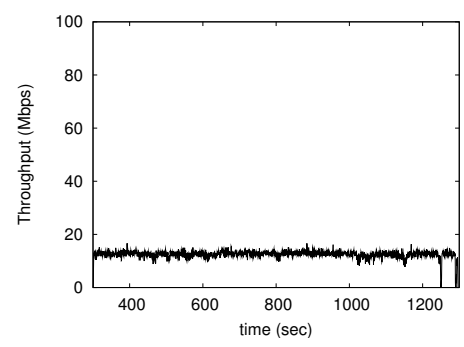

(e) Throughput (FAST transfer)

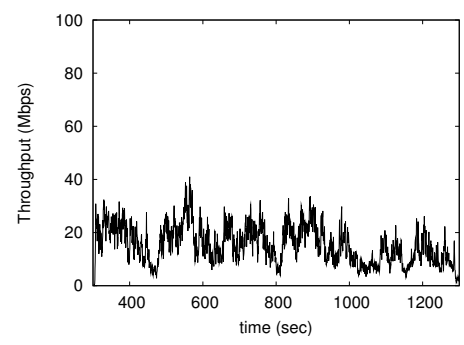

(g) Throughput (RAPID transfer)

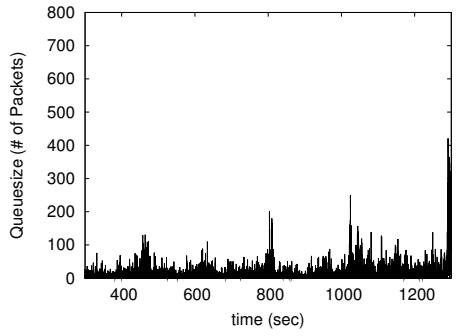

(b) Queue Sizes (TMIX only)

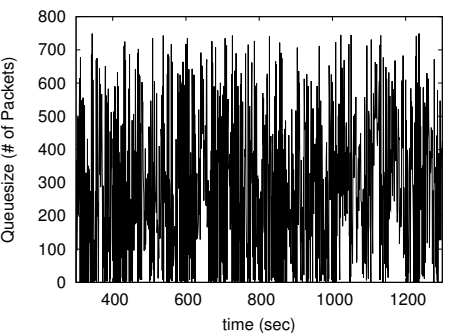

(d) Queue Sizes (HighSpeed+TMIX)

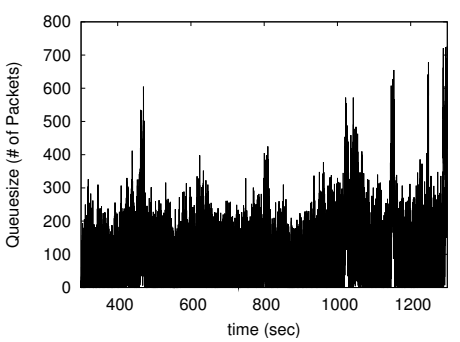

(f) Queue Sizes (FAST+TMIX)

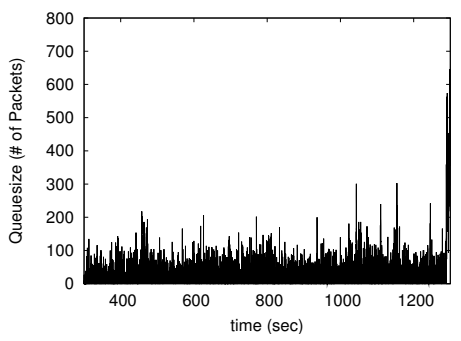

(h) Queue Sizes (RAPID+TMIX)
Fig. 8. Performance with TMIX Cross-traffic

queue buildup by only a small amount. The bottleneck link is nearly-100\% utilized throughout this experiment.

\section{COncluding Remarks and Future Work}

In this paper, we propose a novel protocol, RAPID, that reduces the timescales at which congestion-control operates by orders of magnitude - this enables the protocol to efficiently utilize spare bandwidth in high-speed and dynamic bandwidth environments. RAPID does so while: (i) providing excellent intra-protocol fairness in heterogeneous RTT environments, and (ii) ensuring friendly co-existence with regular TCP transfers and router queues.

Perhaps one of the key challenges to deploying RAPID in multi-gigabit networks is related to the high-precision packet time-stamping and packet-spacing (of the order of a few microseconds) that it would need to rely on. However, note that the RAPID receiver does not directly use packet receive times, but simply relies on detecting increasing trends in the inter-packet gaps - thus requirements on the accuracy of timestamping at the receiver are somewhat relaxed. Furthermore, the inter-packet gaps at the sender can be better controlled by limiting buffering in the network interface card. Consequently, we believe that existing high-end PC platforms should be able to support RAPID for up to 1 Gbps speeds. We are also exploring the use of FPGA-based network cards to enable a RAPID implementation to scale up to multi-gigabit capabilities.

We are currently also conducting a formal analysis of protocol properties such as fairness, efficiency, and stability, especially in scenarios where multiple RAPID senders probe the network concurrently. In addition, we are designing mechanisms to help auto-tune RAPID as it scales up to multi-gigabit and multi-hop networks.

\section{REFERENCES}

[1] M. Allman, V. Paxson, and W. Stevens, "TCP Congestion Control," RFC 2581 (Proposed Standard), Apr. 1999, updated by RFC 3390. [Online]. Available: http://www.ietf.org/rfc/rfc2581.txt

[2] S. Floyd, "HighSpeed TCP for Large Congestion Windows," RFC 3649 (Experimental), Dec. 2003. [Online]. Available: http://www.ietf.org/rfc/ rfc3649.txt

[3] I. Rhee, L. Xu, and S. Ha, "CUBIC for fast long-distance networks," August 2007, Internet Draft.

[4] L. Xu, K. Harfoush, and I. Rhee, "Binary increase congestion control for fast, long distance networks," in Proceedings of IEEE INFOCOM, March 2004.

[5] D. Wei, C. Jin, S. Low, and S. Hegde, "FAST TCP: Motivation, architecture, algorithms, performance," IEEE/ACM Transactions on Networking, vol. 14, no. 6, pp. 1246-1259, 2006.

[6] T. Anderson, A. Collins, A. Krishnamurthy, and J. Zoharjan, "PCP: Efficient endpoint congestion control," in Proceedings of the 3rd Symposium on Networked Systems Design and Implementation (NSDI), May 2006.

[7] M. Fukuhara, F. Hirose, T. Hatano, H. Shigeno, and K. Okada, "SRF TCP: A TCP-friendly and fair congestion control method for high-speed networks," in Proceedings of the International Conference on Principles of Distributed Systems (OPODIS), ser. Lecture Notes in Computer Science, vol. 3544. Springer, 2005, pp. 169-183.

[8] T. Kelly, "Scalable TCP: Improving performance in highspeed wide area networks," in First International Workshop on Protocols for Fast Longdistance Networks, February 2003.

[9] S. Floyd and M. Allman, "Specifying New Congestion Control Algorithms," RFC 5033, 2007. [Online]. Available: http://www.ietf.org/ rfc/rfc5033.txt

[10] V. Ribeiro, R. Riedi, R. Baraniuk, J. Navratil, and L. Cottrell, "pathChirp: Efficient available bandwidth estimation for network paths," in Passive and Active Measurement Workshop, April 2003.

[11] A. Shriram and J. Kaur, "Empirical evaluations of techniques for measuring available bandwidth," in Proceedings of IEEE INFOCOM 2007, May 2007.

[12] V. Konda and J. Kaur, "Shrinking the timescales at which congestioncontrol operates," Technical Report, Department of Computer Science, University of North Carolina at Chapel Hill, August 2008.

[13] S. Floyd, "Limited Slow-Start for TCP with Large Congestion Windows," RFC 3742 (Experimental), Mar. 2004. [Online]. Available: http://www.ietf.org/rfc/rfc3742.txt

[14] S. Gorinsky and H. Vin, "Additive increase appears inferior," Dept. of Computer Sciences, University of Texas at Austin, Tech. Rep., 2000.

[15] M. Vojnovic, J. L. Boudec, and C. Boutremans, "Global fairness of additive-increase and multiplicative- decrease with heterogeneous roundtrip times," in Proceedings of IEEE INFOCOM, March 2000.

[16] "Network simulator-2 ns2 (http://www.isi.edu/nsnam/ns/)."

[17] "URL http://www.cubinlab.ee.unimelb.edu.au/ns2fasttcp/."

[18] R. Jain, D. W. Chiu, and W. R. Hawe, "A quantitative measure of fairness and discrimination for resource alloca tion in shared computer system," September 1984, dEC Research Report TR-301.

[19] M. Weigle, P. Adurthi, F. Hernandez-Campos, K. Jeffay, and F. Smith, "Tmix: A tool for generating realistic application workloads in ns-2," ACM SIGCOMM Computer Communication Review, vol. 26, no. 3, pp.
$67-76,2006$. 